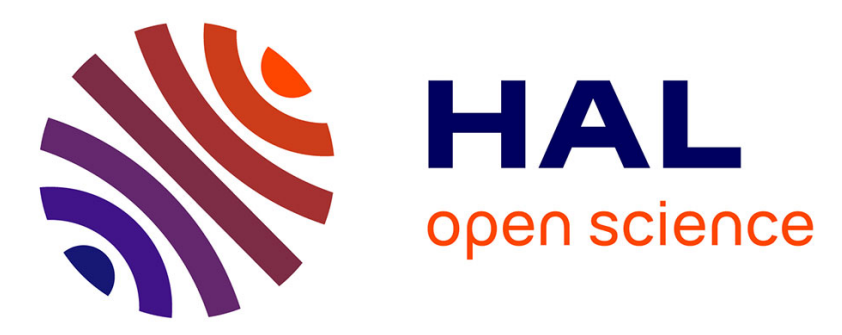

\title{
An imprecision importance measure for uncertainty representations interpreted as lower and upper probabilities, with special emphasis on possibility theory R. Flage, Terje Aven, Piero Baraldi, Enrico Zio
}

\section{- To cite this version:}

R. Flage, Terje Aven, Piero Baraldi, Enrico Zio. An imprecision importance measure for uncertainty representations interpreted as lower and upper probabilities, with special emphasis on possibility theory. Proceedings of the Institution of Mechanical Engineers, Part O: Journal of Risk and Reliability, 2012, 226 (6), pp.656-665. 10.1177/1748006X12467591 . hal-00765296

HAL Id: hal-00765296

https://hal-centralesupelec.archives-ouvertes.fr/hal-00765296

Submitted on 14 Dec 2012

HAL is a multi-disciplinary open access archive for the deposit and dissemination of scientific research documents, whether they are published or not. The documents may come from teaching and research institutions in France or abroad, or from public or private research centers.
L'archive ouverte pluridisciplinaire $\mathbf{H A L}$, est destinée au dépôt et à la diffusion de documents scientifiques de niveau recherche, publiés ou non, émanant des établissements d'enseignement et de recherche français ou étrangers, des laboratoires publics ou privés. 


\title{
An imprecision importance measure for uncertainty representations interpreted as lower and upper probabilities, with special emphasis on possibility theory
}

\author{
R. Flage ${ }^{1}$, T. Aven ${ }^{1}$, P. Baraldi ${ }^{2} \&$ E. Zio ${ }^{3,2}$ \\ ${ }^{1}$ University of Stavanger, Norway \\ ${ }^{2}$ Polytechnic of Milan, Italy \\ ${ }^{3}$ Chair on Systems Science and the Energetic Challenge, European Foundation for New Energy-Electricité de \\ France, EcoleCentrale Paris and Supelec, France
}

Uncertainty importance measures typically reflect the degree to which uncertainty about risk and reliability parameters at the component level influences uncertainty about parameters at the system level. The definition of these measures is typically founded on a Bayesian perspective where subjective probabilities are used to express epistemic uncertainty; hence, they do not reflect the effect of imprecision in probability assignments, as captured by alternative uncertainty representation frameworks such as imprecise probability, possibility theory and evidence theory. In the present paper, we define an imprecision importance measure to evaluate the effect of removing imprecision to the extent that a probabilistic representation of uncertainty remains, as well as to the extent that no epistemic uncertainty remains. Possibility theory is highlighted throughout the paper as an example of an uncertainty representation reflecting imprecision, and used in particular in two numerical examples which are included for illustration.

Key words: imprecision; importance measure; epistemic uncertainty 
The use of importance measures (IM) is an integral part of reliability and risk analysis. IM are used to study the effect on system level reliability or risk parameters of altering component level parameters. A number of uncertainty importance measures (UIM) have also been proposed in the literature (Aven \&Nøkland, 2010). These extend the 'classical' reliability and risk IM in the presence of epistemic uncertainty. UIM are used to study to what degree uncertainty about risk and reliability parameters at the component level influences uncertainty about parameters at the system level.

In general terms, we are interested in the quantity $\mathrm{Y}$, possibly a vector, and introduce a model $\mathrm{g}(\mathrm{X})$ which relates $\mathrm{n}$ input quantities $\mathrm{X}=\left(\mathrm{X}_{1}, \mathrm{X}_{2}, \ldots, \mathrm{X}_{\mathrm{n}}\right)$ to the output quantity of interest $\mathrm{Y}$. In particular,we are interested in an output quantity $\mathrm{Y}=\mathrm{p}=\mathrm{g}(\mathrm{q})$, function of the input $\mathrm{X}=\mathrm{q}$ where $\mathrm{p}$ and $\mathrm{q}$ are reliability or risk parameters at the system and component level, respectively. Typically, $\mathrm{p}$ and $\mathrm{q}$ are given the interpretation of long-run frequencies, e.g. the fraction of time a system and its components are functioning, respectively. This is the interpretation adopted, for example, in the probability of frequency approach to risk analysis (Kaplan \& Garrick, 1981).

Classical IM are used to analyze changes to $\mathrm{p}$ given changes to q. For example, the so-called 'improvement potential' of component $i$ is defined as the change to the system availability $p$ when the component availability $\mathrm{q}_{\mathrm{i}}$ is set equal to 1 , and the Birnbaum IM is defined as the partial derivative of $\mathrm{p}$ with respect to $\mathrm{q}_{\mathrm{i}}$ (e.g. Aven \& Jensen, 1999; Rausand\&Høyland, 2004).

UIM are typically founded on a Bayesian perspective. A subjective probability distribution F is introduced for $\mathrm{q}$ and propagated through a model $\mathrm{g}$. The result is a probability distribution of $\mathrm{p}$, and UIMs are used to analyse changes to the distribution of $\mathrm{p}$ given changes to $\mathrm{F}$. Reference is made to Section 2 for a brief review of IM and UIM.

In a Bayesian perspective subjective probabilities express epistemic uncertainty; hence, they do not reflect imprecision in probability assignments. The term imprecision here labels the phenomenon captured by a wide range of extensions of the classical theory of probability, including lower and upper pre-visions (Walley, 1991), belief and plausibility functions 
(Dempster, 1967; Shafer, 1976), possibility measures (Dubois \&Prade, 1988), fuzzy sets (Zadeh, 1965), robust Bayesian methods (Berger, 1984), p-boxes (Ferson et al., 2003) and interval probabilities (Weichselberger, 2000).

One much studied type of UIM is that reflecting the effect on system level parameter uncertainty of removing component level parameter uncertainty. For example, for a probability distribution $\mathrm{F}$ of component level parameters $\mathrm{q}$ which propagated through a model $\mathrm{g}$ induces a probability distribution of the system level parameter $\mathrm{p}$, this type of UIM evaluates changes to the distribution of $\mathrm{p}$ by assuming $\mathrm{q}_{\mathrm{i}}$ known for some $\mathrm{i}$. Of course, the value of $\mathrm{q}_{\mathrm{i}}$ cannot be specified with certainty and so the resulting measure becomes a function of $q_{i}$. An example is the measure $\operatorname{Var}(p)-\operatorname{Var}\left(p \mid q_{i}\right)$, expressing the reduction in the variance of the system level parameter $\mathrm{p}$ that is achieved by specifying the value of the component level parameter $\mathrm{q}_{\mathrm{i}}$. One way to proceed is to consider the expected value of the above measure, namely (Iman, 1987):

$$
\operatorname{Var}(p)-E\left[\operatorname{Var}\left(p \mid q_{i}\right)\right]=\operatorname{Var}\left(E\left[p \mid q_{i}\right]\right)
$$

Aven \& Nøkland (2010) investigate the link between UIM and traditional IM. In doing so they distinguish between the cases that $\mathrm{X}$ and $\mathrm{Y}$, as introduced above, are (a) observable events and quantities, such as the occurrence of a system failure and the number of system failures, and (b) unobservable parameters, such as p and q. Based on their findings a combined set of IM and UIM is defined.

Within a non-probabilistic framework, a Fuzzy Uncertainty Importance Measure (FUIM) has been proposed in (Suresh et al., 1996) to identify those component level parameters $\mathrm{q}_{\mathrm{i}}$ having the greatest impact on the uncertainty of the system level parameter p. The FUIM measures the distance between the output fuzzy sets considering the input parameters $\mathrm{q}_{\mathrm{i}}$ with or without uncertainty. In (Baraldi et al., 2009), the FUIM has been modified in order to consider the different imprecision in the output fuzzy sets, measured in terms of fuzzy specificity, instead of the difference between the fuzzy sets. In (Liping \& Fuzheng, 2009), an importance measure based on the concept of possibilistic entropy has been proposed and applied to fault tree analysis in a possibilistic framework. 
In the present paper, we consider the case that a distribution pair $\mathrm{H}_{\mathrm{q}}$ is introduced for $\mathrm{q}$. We may for example have $\mathrm{H}_{\mathrm{q}}=\left[\mathrm{N}_{\mathrm{q}}, \Pi_{\mathrm{q}}\right]$, where $\mathrm{N}_{\mathrm{q}}$ and $\Pi_{\mathrm{q}}$ are the cumulative necessity and possibility distributions (from possibility theory) of $\mathrm{q}$, respectively; or $\mathrm{H}_{\mathrm{q}}=\left[\mathrm{Bel}_{\mathrm{q}}, \mathrm{Pl}_{\mathrm{q}}\right]$, where Bel $_{\mathrm{q}}$ and $\mathrm{Pl}_{\mathrm{q}}$ are the cumulative belief and plausibility distributions (from evidence theory) of $\mathrm{q}$, respectively; or $\mathrm{H}_{\mathrm{q}}=\left[\mathrm{H}_{\mathrm{q}}{ }^{1}, \mathrm{H}_{\mathrm{q}}{ }^{\mathrm{u}}\right]$ where $\mathrm{H}_{\mathrm{q}}{ }^{1}$ and $\mathrm{H}_{\mathrm{q}}{ }^{\mathrm{u}}$ are lower and upper imprecise probability distributions of $\mathrm{q}$, respectively. In the present paper, possibility theory is highlighted throughout the paper as an example of an uncertainty representation reflecting imprecision. The choice of possibility theory in this early study of the suggested IIM is due to its mathematical simplicity; cf. Dubois (2006) who notes that 'Possibility theory is one of the current uncertainty theories devoted to the handling of incomplete information, more precisely it is the simplest one, mathematically'.

Defining the imprecision of a distribution pair as the area between its lower and upper cumulative distributions, we define an imprecision importance measure (IIM) that evaluates the effect on system level parameter imprecision of removing component level parameter imprecision. Two extents of imprecision removal are possible:

i. Removal of imprecision to the extent that a probabilistic representation remains

ii. Removal of imprecision to the extent that no epistemic uncertainty remains

The latter case may be seen as a special case of the former. The definition of an IIM in terms of imprecision removal is associated with an analogous problem as was seen above for uncertainty removal in the case of UIM; namely, the measure can be defined but neither the specific value of a component level parameter nor its probability distribution can really be specified. We are led to consider, respectively:

I. A probability distribution consistent with $\mathrm{H}_{\mathrm{q}}$

II. The IIM as a function of $\mathrm{q}_{\mathrm{i}}$

In the following we refer to these as type I and type II measures. Flage et al. (2011) study the type II measure. In the present paper,we extend the work of Flage et al. (2011) and study also the type I measure in the case that $\mathrm{H}_{\mathrm{q}}=\left[\mathrm{N}_{\mathrm{q}}, \Pi_{\mathrm{q}}\right]$. A probability distribution is obtained from $\mathrm{H}_{\mathrm{q}}$ by considering a possibility-probability transformation procedure, and further computations take place within the framework of a hybrid probabilistic/possibilistic method. 
The remainder of the paper is organized as follows: In Section 2, we review some basic classical IM and some UIM. In Section 3, we review the concepts of uncertainty and imprecision, as well as their representation. In Section 4, we define an IIM as indicated above, and in Section 5 the suggested measure is evaluated in terms of a numerical example where possibility theory is used as the representation of uncertainty. Section 6 provides a discussion and some conclusions.

\section{Importance measures and uncertainty importance measures}

There are essentially two fundamental classical importance measures: the 'improvement potential' of a component, describing the effect on the system reliability of making the component perfectly reliable; the Birnbaum importance measure, reflecting the effect on system reliability of an incremental change in the reliability of a component. The improvement potential of a component is defined by (e.g. Aven \& Jensen, 1999; Rausand \& Høyland, 2004)

$$
h\left(1_{i}, q\right)-h(q)
$$

where $h(q)$ is the system reliability function expressing $p$ as a function of $q$; and $h\left(1_{i}, q\right)=$ $\mathrm{h}\left(\mathrm{q}_{1}, \ldots, 1_{\mathrm{i}}, \ldots \mathrm{q}_{\mathrm{n}}\right)$ the system reliability function when component $\mathrm{i}$ is perfectly reliable. The importance measures referred to as risk achievement worth (RAW) and risk reduction worth (RRW) (e.g. Cheok et al., 1998; Rausand\&Høyland, 2004; Zio, 2009) represent minor adjustments of the improvement potential importance measure. The Birnbaum importance measure is defined by (e.g. Aven \& Jensen, 1999; Rausand \& Høyland, 2004; Zio, 2009)

$$
\frac{\partial h(q)}{\partial q_{i}}
$$

i.e. as the partial derivative of the system reliability with respect to $\mathrm{q}_{\mathrm{i}}$. The improvement potential importance measure is most relevant in the design phase of a system, whereas the 
Birnbaum importance measure is most relevant in the operational phase (Aven\& Jensen, 1999). See Rausand \& Høyland (2004) and Zio (2009) for a more in-depth review of classical IMs.

Uncertainty importance measures were described to some extent in Section 1. The UIM by Iman (1987) is variance-based and hence an example of a measure in one of the three categories described by Borgonovo (2006):

i. Non parametric techniques (input-output correlation)

ii. Variance-based importance measures

iii. Moment-independent sensitivity indicators.

See Borgonovo (2006) for a more in-depth review of UIMs.

\section{Uncertainty, imprecision and its representation}

In engineering risk analysis a distinction is commonly made between aleatory (stochastic) and epistemic (knowledge-related) uncertainty (e.g. Apostolakis, 1990; Helton \& Burmaster, 1996). Aleatory uncertainty refers to variation in populations. Epistemic uncertainty refers to lack of knowledge about phenomena and usually translates into uncertainty about the parameters of a model used to describe random variation. Whereas epistemic uncertainty can be reduced, aleatory uncertainty cannot and for this reason it is sometimes called irreducible uncertainty (Helton \& Burmaster, 1996).

Traditionally, limiting relative frequency probabilities are used to describe aleatory uncertainty and subjective probabilities are used to describe epistemic uncertainty. However, as described in Section 1, several alternatives to probability as representation of epistemic uncertainty have been suggested, the motivation being to capture imprecision in subjective probability assignments. Imprecision here refers to inability to precisely specify a probability (distribution). Presumably an analyst/expert would ideally want to characterize epistemic uncertainty using a subjective probability (distribution); however, due to limitations in the information available (e.g. lack of data, lack of phenomenological understanding) the analyst/expert is unable or not willing to specify a single subjective probability (distribution) 
and only able to or willing to specify a probability interval (a family of probability distributions).

For example, numerical possibility distributions can encode special convex families of probability measures (Dubois, 2006). In possibility theory, uncertainty and imprecision is represented by a possibility function $\pi$. For each element $\omega$ in a set $\Omega, \pi(\omega)$ expresses the degree of possibility of $\omega$. Since one of the elements of $\Omega$ is the true value, it is assumed that $\pi(\omega)=1$ for at least one element $\omega$. The possibility measure of an event $A, \Pi(A)$, is defined by

$$
\Pi(A)=\sup _{\omega \in \Omega} \pi(\omega)
$$

and the necessity measure of $\mathrm{A}, \mathrm{N}(\mathrm{A})$, by

$$
\mathrm{N}(\mathrm{A})=1-\Pi(\bar{A})
$$

Uncertainty about the occurrence of an event A, then, is represented by the pair [N(A), П(A)], where the necessity and possibility measures can be given the interpretation of lower and upper probabilities induced from specific convex sets of probability functions (Dubois, 2006):

$$
\mathcal{P}(\pi)=\{P: \forall A \text { measurable, } N(A) \leq P(A)\}=\{P: \forall A \text { measurable, } P(A) \leq \Pi(A)\} .
$$

Then, $\sup _{P \in \mathcal{P}(\pi)} P(A)=\Pi(A)$ and $\inf _{P \in \mathcal{P}(\pi)} P(A)=\mathrm{N}(A)$ (see e.g. Dubois \& Prade, 1992).

Another point of view on possibility theory is a graded view where possibility measures express the extent to which an event is plausible, i.e. consistent with a possible state of the world, and necessity measures express the certainty of events. Reference is made to Dubois (2006) and the references therein.

\section{$4 \quad$ An imprecision importance measure}


Consider the system level reliability or risk parameter $\mathrm{p}$ and its distribution pair $\mathrm{H}_{\mathrm{p}}$ induced by the propagation of the distribution pair $\mathrm{H}_{\mathrm{q}}$ for a set of lower level parameters $q$ through a model g. Define the imprecision of a distribution pair $\mathrm{H}$, denoted $\Delta \mathrm{H}$, as the area between its lower and upper cumulative distributions, i.e.

$$
\Delta H=\int(\max H(x)-\min H(x)) d x
$$

as illustrated in Figure 1.

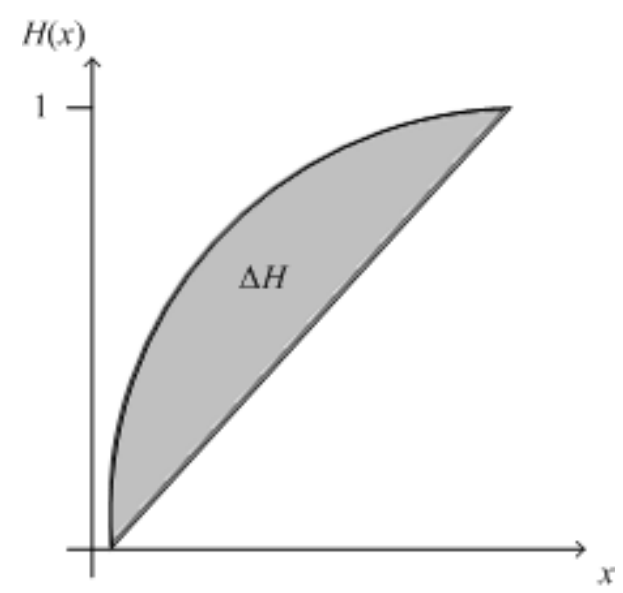

Figure 1.Imprecision measure $\Delta \mathrm{H}$ equal to the area between the lower and upper distributions in the distribution pair $\mathrm{H}_{\mathrm{p}}$.

For example, in the case of a distribution pair $\mathrm{H}=[\mathrm{N}, \Pi]$ induced by a triangular possibility distribution $\pi$ with support $S$, we have - by geometrical considerations and recalling that a possibility distribution has unit height - that the imprecision of the possibility distribution is $\Delta(\mathrm{H})=|\mathrm{S}| / 2$. In the case of a probabilistic representation of uncertainty we have $\max \mathrm{H}(\mathrm{x})=$ $\min \mathrm{H}(\mathrm{x})$ for all $\mathrm{x}$, and hence $\Delta \mathrm{H}=0$.

Now define $\Delta_{\mathrm{i}}\left(\mathrm{H}_{\mathrm{p}}\right)$ as the imprecision of $\mathrm{H}_{\mathrm{p}}$ when the imprecision of the distribution on the parameter $\mathrm{q}_{\mathrm{i}}$ is removed. We may then define an imprecision removal importance measure (IRIM) as

$$
I_{i}=\Delta H_{p}-\Delta_{\mathrm{i}} H_{p}
$$


which expresses the amount of system level imprecision removal that comes from removing imprecision at the component level. The relative imprecision removal effect can be studied in terms of the measure

$$
\bar{I}_{i}=\frac{\Delta H_{p}-\Delta_{\mathrm{i}} H_{p}}{\Delta H_{p}}
$$

which expresses the fraction of imprecision associated with the distribution pair $\mathrm{H}_{\mathrm{p}}$ that is attributable to component i.

As described in Section 1, imprecision can be removed either to the extent that a probabilistic representation remains, or to the extent that no epistemic uncertainty remains.

\subsection{Type I measure}

Removal of imprecision to the extent that a probabilistic representation remains means that uncertainty about $q_{i}$ is described using a (subjective) probability distribution $F_{i}(x)=P\left(q_{i} \leq x\right)$, as illustrated in Figure 2.

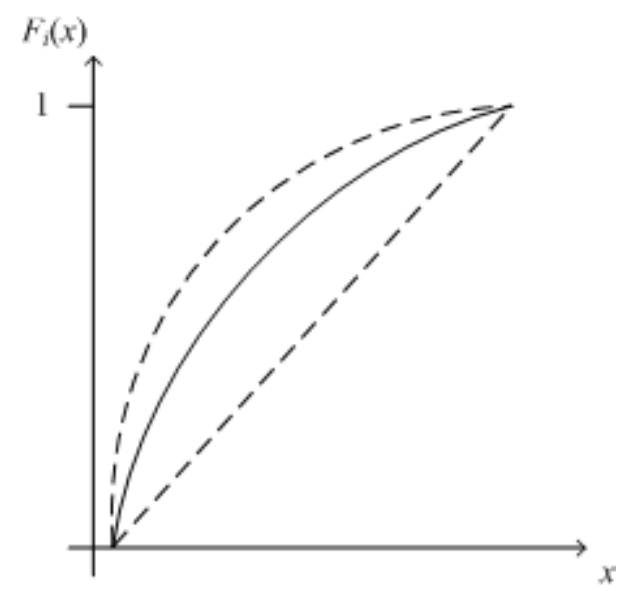

Figure 2.Removal of imprecision (imprecise probability distribution - dashed lines) to the extent that a singlevalued probabilistic representation remains (solid line). 
A probability distribution can be derived from a possibility distribution by considering a possibility-probability transformation procedure. Then a hybrid probabilistic/possibilistic method can be used for the joint propagation of the resulting probability distribution together with the remaining possibility distributions. In the following we review a possibilityprobability transformation procedure based on Dubois et al. (1993) and a hybrid probabilistic/possibilistic method based on Baudrit et al. (2006) and applied in the context of risk analysis by (Baraldi\&Zio, 2008).

\subsubsection{Possibility-probability transformation procedure}

Possibility-probability transformations (as well as probability-possibility transformations) are based on given principles and ensure a consistent transformation to the extent that there is no violation of the formal rules (definitions) connecting probability and possibility when possibility and necessity measures are taken as upper and lower probabilities, and so that the transformation is not arbitrary within the constraints of these rules. Nevertheless, as noted in (Dubois et al., 1993):

... going from a probabilistic representation to a possibilistic representation, some information is lost because we go from point-valued probabilities to interval-valued ones; the converse transformation adds information to some possibilistic incomplete knowledge. This additional information is always somewhat arbitrary.

When possibilityand necessity measures are interpreted as upper and lower probabilities, a possibility distribution $\pi$ can be seen as inducingthe family $\mathcal{P}(\pi)$ Pdefined in Equation (6) of probability measures. Since there is not a one-to-one relation between possibility and probability, a transformation from a possibility distribution $\pi$ into a probability measure $\mathrm{P}$ can only ensure that

a) $\mathrm{P}$ is a member of $\mathcal{P}(\pi) \mathbf{P}$

b) $\mathrm{P}$ is selected among the members of $\mathcal{P}(\pi)$ Paccording to some principle (rationale); e.g. 'minimize the information content of $\mathrm{P}^{\prime}$, in some sense

Various possibility-probability and probability-possibility transformations have been suggested in the literature.The principle of insufficient reason specifies that maximum 
uncertainty on an interval should be described by a uniform probability distribution on that interval. A sampling procedure for transforming a possibility distribution $\pi$ into a probability distribution $\mathrm{P}$ according to the principle of insufficient reason is:

- Sample a random value $\alpha^{*}$ in $(0,1]$ and consider the $\alpha$-cut level $\mathrm{L}_{\alpha^{*}}=\left\{\mathrm{x}: \pi(\mathrm{x}) \geq \alpha^{*}\right\}$

- Sample $\mathrm{x}^{*}$ at random in $\mathrm{L}_{\alpha^{*}}$

The probability density $\mathrm{f}$ resulting from a transformation of $\pi$ is given by

$$
f(x)=\int_{0}^{\pi(x)} \frac{d \alpha}{\left|L_{\alpha}\right|}
$$

where $\left|L_{\alpha}\right|$ is the length of the alpha-cut levels of $\pi$. To motivate this, note that

$$
f(x)=\int_{0}^{1} f(x \mid \alpha) f(\alpha) d \alpha
$$

From step 1 in the sampling procedure above we have $f(\alpha)=1$, and from step 2 that

$$
f(x \mid \alpha)=\frac{1}{\left|L_{\alpha}\right|}
$$

For the integration space we note that $f(x \mid \alpha)=0$ for $\alpha>\pi(x)$. The densityf is the centre of gravity of $\mathcal{P}(\pi) \mathbf{P}$. The transformation in Equation (10) applies to upper semi-continuous, unimodal possibility distributions $\pi$ with bounded support.

Another possibility to probability transformation principle, based on maximum entropy, consists in selecting the $\mathrm{P}$ in $\mathcal{P}(\pi)$ which maximizes entropy. In general, however, this transformation violates the preference preservation constraint (Dubois et al., 1993) and is as such less attractive. 


\subsubsection{Hybrid combination procedure}

By the hybrid procedure (Baudrit et al., 2006), propagation of uncertainty is based on a combination of the Monte Carlo technique (e.g. Kalos\& Whitlock, 1986) and the extension principle of fuzzy set theory (e.g. Zadeh, 1965). The main steps of the procedure are:

- Repeated Monte Carlo samplings of the probabilistic quantities

- Fuzzy interval analysis of the possibilistic quantities

Considering the functional relationship $\mathrm{p}=\mathrm{g}(\mathrm{q})$ studied in the present paper, the transformation procedure described in the preceding Section leads to a situation where uncertainty related to a single parameter $\mathrm{q}_{\mathrm{i}}$ is described by a probability distribution $\mathrm{F}_{\mathrm{i}}$, while uncertainty related to the remaining $\mathrm{n}-1$ parameters are described by possibility distributions $\left(\pi_{1}, \ldots, \pi_{\mathrm{i}-1}, \pi_{\mathrm{i}+1}, \ldots \pi_{\mathrm{n}}\right)$. For a fixed value of $\mathrm{q}_{\mathrm{i}}$, obtained by Monte Carlo sampling, the extension principle defines the possibility distribution of $\mathrm{p}$ as

$$
\pi_{p}(x)=\sup _{q, g(q)=p} \min \left\{\pi_{1}, \ldots, \pi_{i-1}, \pi_{i+1}, \ldots, \pi_{n}\right\}
$$

We take $\mathrm{m}=10^{4}$ Monte Carlo samplings and determine the imprecision reduction from the transformation from $\pi_{\mathrm{i}}$ to $\mathrm{F}_{\mathrm{i}}$ as the average imprecision reduction.

\subsection{Type II measure}

Removal of imprecision to the extent that no epistemic uncertainty remains means that $\mathrm{q}_{\mathrm{i}}$ can be specified with certainty, and the uncertainty hence represented by the step function $u_{i}(x)$, where $u_{i}(x)$ is equal to 0 for $x<q_{i}$ and equal to 1 for $x \geq q_{i}$, as illustrated in Figure 3 . 


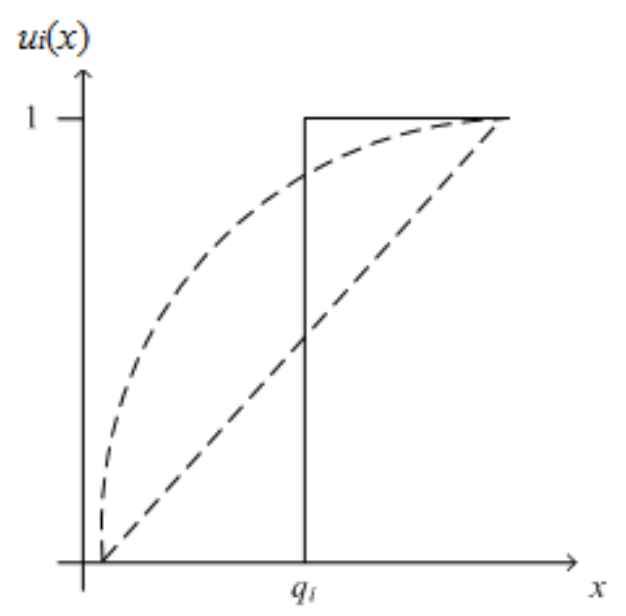

Figure 3.Removal of imprecision (imprecise probability distribution - dashed lines) to the extent that a no epistemic uncertainty remains (solid line).

In the case of removal of imprecision to the extent that no imprecision remains, we are led to consider the suggested imprecision importance measure as a function of $\mathrm{q}_{\mathrm{i}}$, denoted $\mathrm{I}_{\mathrm{i}}{ }_{\mathrm{i}}\left(\mathrm{q}_{\mathrm{i}}\right)$.

Section 5 presents a numerical example evaluating type I and type II measures.

\subsection{Imprecision importance measures in presence of dependences}

Future work will be devoted to the investigation of the proposed imprecision uncertainty importance measures in presence of dependences in the input considered for the analysis. In practice, two types of dependencies may need to be considered: i) epistemic dependence between the uncertainty on the component parameters and ii) physical dependence between the system components. The former case relates to situations in which the information on the values of the parameters of two or more system components is correlated. For example, if there are two identical components in the system and the same information is used to estimate their characteristic parameters, then the uncertainty on them will be the same and identically represented. In this case, the procedures of uncertainty removal should be modified in order to consider that the reduction of the uncertainty on a single component parameter can cause the (same) reduction of the uncertainty on other correlated parameters. Contrarily, the physical dependence between the system components is not expected to influence the procedures of uncertainty removal, since this dependence has an effect on the aleatory character of the modeled process but not on the epistemic uncertainty on the component parameters. 
On the contrary, the procedure for the propagation of the uncertainty from the component level parameters (input quantities) to the system level parameter (output quantity) should be modified in both cases of dependence. On this subject, the interested reader may refer to Pedroni and Zio (2012).

\section{$5 \quad$ Numerical example}

Consider a system $\mathrm{S}$ consisting of five independent components connected asillustrated by the reliability block diagram in Figure4.

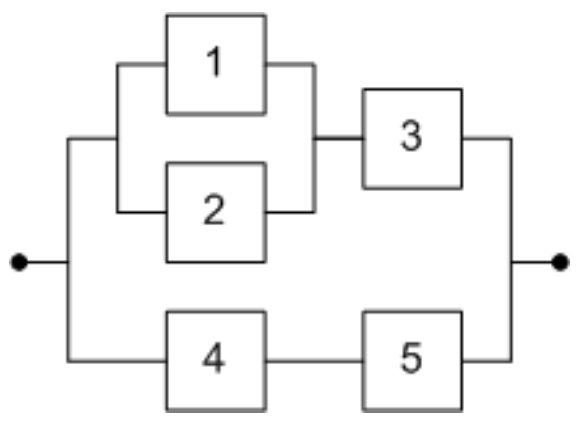

Figure 4. Reliability block diagram of system S.

Component $i$ has availability $q_{i}, i=1,2,3$. The availability of the system, denoted $p$, can then be expressed as

$$
p=1-\left(1-\left(1-\left(1-q_{1}\right)\left(1-q_{2}\right)\right) q_{3}\right)\left(1-q_{4} q_{5}\right)
$$

The component availability parameters $\mathrm{q}=\left(\mathrm{q}_{1}, \mathrm{q}_{2}, \mathrm{q}_{3}, \mathrm{q}_{4}, \mathrm{q}_{5}\right)$ are assumed to be unknown, the uncertainty being described using marginal necessity/possibility distribution pairs $\mathrm{H}=\left(\mathrm{H}_{1}, \mathrm{H}_{2}, \mathrm{H}_{3}, \mathrm{H}_{4}, \mathrm{H}_{5}\right)$, where $\mathrm{H}_{\mathrm{i}}(\mathrm{x})=\left[\mathrm{N}\left(\mathrm{q}_{\mathrm{i}} \leq \mathrm{x}\right), \Pi\left(\mathrm{q}_{\mathrm{i}} \leq \mathrm{x}\right)\right], \mathrm{i}=1,2,3,4$, 5.Due to the restrictions in terms of the type of possibility distributions that the transformation method described in Section 4.1.1 applies to, only triangular possibility distributions will be considered in relation to the type I measure in Section 5.1. In Section 5.2 also trapezoidal and uniform possibility distributions are considered in relation to the type II measure.

\subsection{Type I measure}


We assume that the distributions on the component availabilities and the resulting distribution on the system availability are asshown in Figure 5.
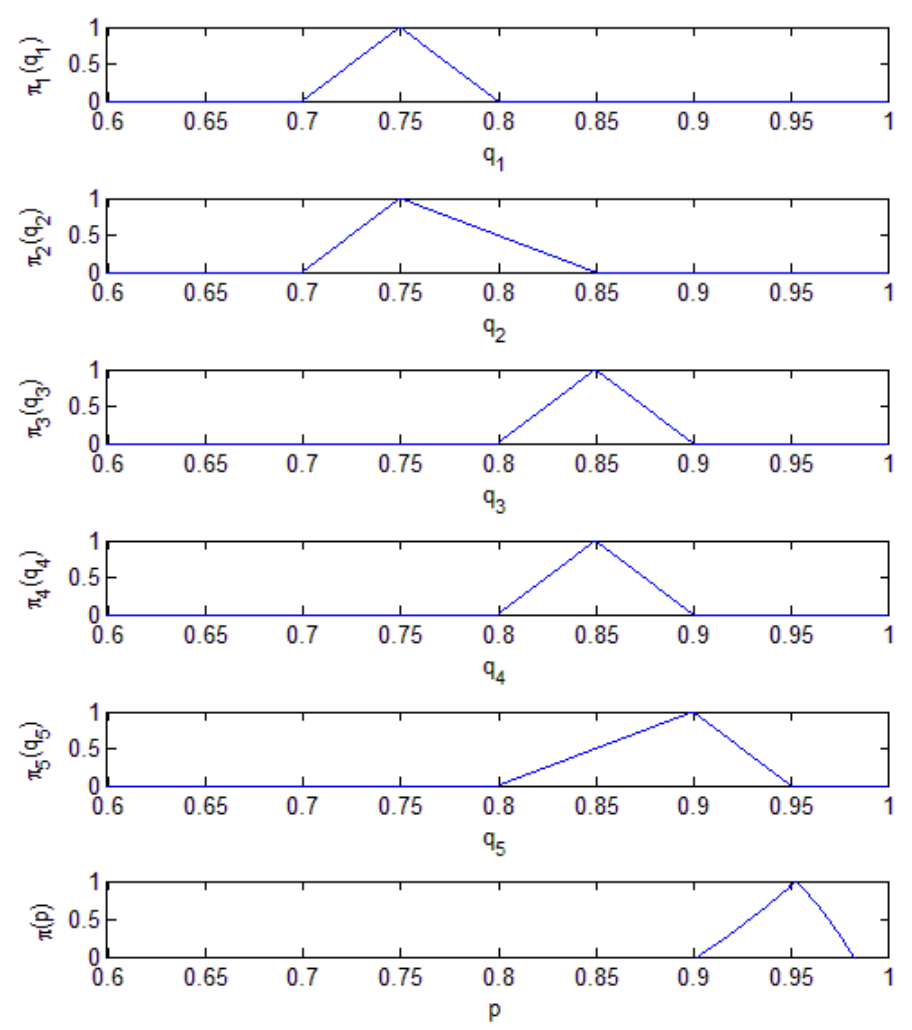

Figure 5. Input distribution functions on component availabilities and resulting system availability.

Let $s_{1}, s_{2}$ and $c$ denote the lower support limit,the upper support limit and the core value of a triangular possibility distribution, respectively. For this type of distribution the imprecision equals

$$
\Delta H=\frac{s_{2}-s_{1}}{2}
$$

Table 1 lists the possibility distribution parameters and the associated imprecision at both component and system level. The imprecision related to the resulting distribution for the system availability is determined as the Riemann sum over $\mathrm{c}=10^{3} \alpha$-cuts. 
Table 1.Component and system availability distribution parameters and imprecision for system S.

\begin{tabular}{lllll}
\hline $\mathrm{i}$ & $\mathrm{s}_{1}$ & $\mathrm{c}$ & $\mathrm{s}_{2}$ & \multicolumn{1}{l}{$\Delta \mathrm{H}$} \\
\hline 1 & & & & \\
2 & 0.70 & 0.75 & 0.80 & 0.050 \\
3 & 0.70 & 0.75 & 0.85 & 0.075 \\
4 & 0.80 & 0.85 & 0.90 & 0.050 \\
5 & 0.80 & 0.85 & 0.90 & 0.050 \\
& 0.80 & 0.90 & 0.95 & 0.075 \\
System & 0.90 & 0.95 & 0.98 & 0.040 \\
& & & & \\
\hline
\end{tabular}

Table 2 summarises the values of the type I imprecision importance measure. The imprecision importance ranking is $[5,3,4,2,1]$.

Table 2.Type I imprecision importance value ranges.

\begin{tabular}{lll}
\hline $\mathrm{i}$ & $I_{i}^{I}$ & $\bar{I}_{i}^{I}$ \\
\hline 1 & 0.0026 & $6.48 \%$ \\
2 & 0.0039 & $9.89 \%$ \\
3 & 0.0116 & $29.1 \%$ \\
4 & 0.0087 & $22.0 \%$ \\
5 & 0.0129 & $32.4 \%$ \\
\hline
\end{tabular}

Notice that although components 2 and 5 are characterized by the same imprecision $\Delta \mathrm{H}=$ 0.075, component 2 imprecision importance measure is lower than that of component 5 due to their different position in the system block diagram. In particular, since component 2 is in parallel to component 1 , a failure of component 2 does not cause the unavailability of the upper system branch. Thus, component 2 has a lower impact on the system unavailability imprecision than component 5 whose failure would cause the unavailability of the bottom system branch. 


\section{$5.2 \quad$ Type II measure}

We now assume that the distributions on thecomponent availabilities and the resulting distribution on the system availability are asshownin Figure 7.
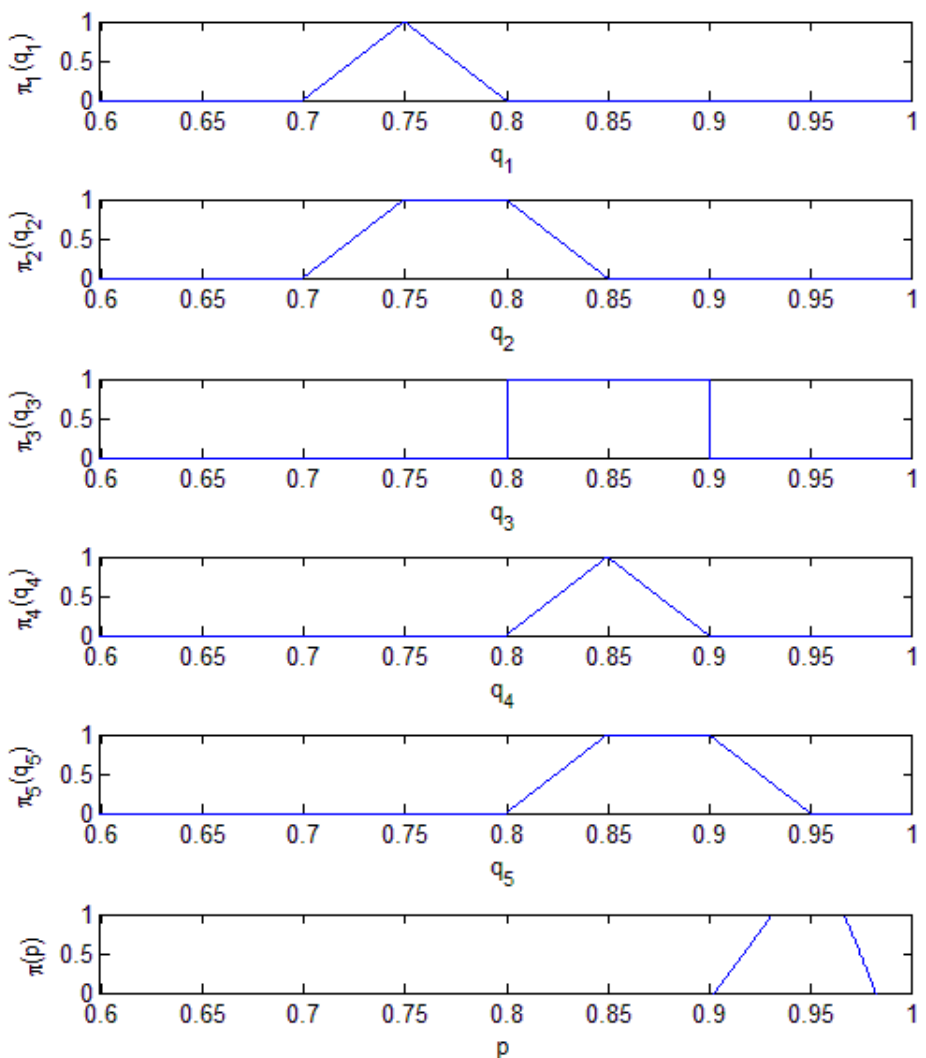

Figure 7. Input distribution functions on component availabilities and resulting system availability.

Let $s_{1}$ and $s_{2}\left(c_{1}\right.$ and $\left.c_{2}\right)$ denote the lower and upper support (core) limits of a possibility distribution, respectively. For a trapezoidal distribution we have $s_{1}<c_{1}<c_{2}<s_{2}$, for a triangular distribution $\mathrm{s}_{1}<\mathrm{c}_{1}=\mathrm{c}_{2}<\mathrm{s}_{2}$, and for a uniform distribution $\mathrm{s}_{1}=\mathrm{c}_{1}<\mathrm{c}_{2}=\mathrm{s}_{2}$. For these distribution classes we then have that the imprecision equals

$$
\Delta H=\frac{s_{2}-s_{1}+c_{2}-c_{1}}{2} .
$$


Table 1 lists the possibility distribution parameters and the associated imprecision, at both component and system level.

Table 3.Component and system availability distribution parameters and imprecision for system S.

\begin{tabular}{llllll}
\hline $\mathrm{i}$ & $\mathrm{s}_{1}$ & $\mathrm{c}_{1}$ & $\mathrm{c}_{2}$ & $\mathrm{~s}_{2}$ & $\Delta \mathrm{H}_{\mathrm{q}}$ \\
\hline 1 & & & & & \\
\hline 2 & 0.70 & 0.75 & 0.75 & 0.80 & 0.05 \\
3 & 0.70 & 0.75 & 0.80 & 0.85 & 0.10 \\
4 & 0.80 & 0.80 & 0.90 & 0.90 & 0.10 \\
5 & 0.80 & 0.85 & 0.85 & 0.90 & 0.05 \\
\hline System & 0.80 & 0.85 & 0.90 & 0.95 & 0.10 \\
& & & & & \\
\hline
\end{tabular}

Figure 8shows the relative variant of the type II imprecision importance measure for all five components in system $\mathrm{S}$. 

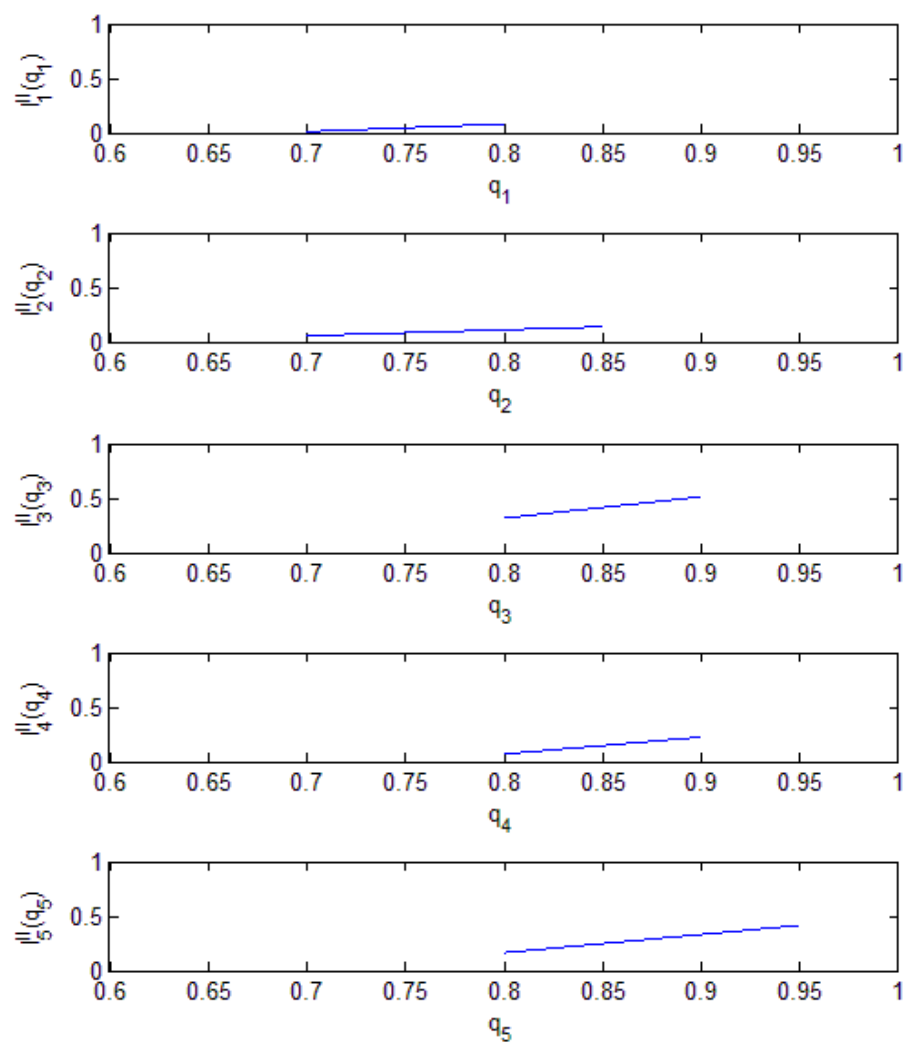

Figure 8.Relative variant of the type II imprecision importance measurefor each component insystem S.

The (relative) imprecision importance for each component is evaluated as a function of $\mathrm{q}_{\mathrm{i}}$ on the support of the associated distribution. Table 2 summarises the value ranges of the (R)IRIM. The imprecision importance ranking is $[3,5,4,2,1]$ according to both high and low values.

Table 4.Type II imprecision importance value ranges.

$\mathrm{i} \quad I_{i}^{I I} \quad \bar{I}_{i}^{I I}$

$1 \quad[0.0006,0.0046] \quad[1.13 \%, 8.10 \%]$

$2 \quad[0.0032,0.0079] \quad[5.60 \%, 13.8 \%]$

$3 \quad[0.0182,0.0294] \quad[31.7 \%, 51.2 \%]$

$4 \quad[0.0040,0.0129] \quad[6.94 \%, 22.5 \%]$

$5 \quad[0.0094,0.0239] \quad[16.4 \%, 41.6 \%]$ 
Notice that, although components 1 and 2 are in parallel, component 2 is characterized by larger Type II imprecision importance value ranges than component 1 . This is due to the fact that our knowledge on $\mathrm{q}_{2}$ is more imprecise than that on $\mathrm{q}_{1}$, being $\Delta \mathrm{H}_{2}=0.10$ whereas $\Delta \mathrm{H}_{1}=$ 0.05. Thus, as expected, removing the imprecision on the more imprecise input causes a larger reduction of the system unavailability imprecision.

\section{$6 \quad$ Discussion and conclusions}

In the present paper, we have described and applied an importance measure that can be used to evaluate the effect on system level parameter imprecision of removing component level parameter imprecision. Hence, the suggested measure is defined analogously to the classical improvement potential IM which describes the effect of removing the unreliability of a component, and analogously with a number of UIMs that describe the effect of removing uncertainty about component performance.

Two extents of imprecision removal are considered: reduction to a probabilistic representation (type I) and removal of epistemic uncertainty (type II), the latter a special case of the former.

The relative version of the measure expresses the fraction of the initial amount of imprecision on the system level parameter that is attributable to each component. In a ranking setting this format is perhaps easier to comprehend than the underlying absolute numbers; however, the fractions need to be seen in relation to the initial amount of imprecision on the system level parameter.

IIMs may be seen as an extension of UIMs when the uncertainty representation is no longer single-valued probability but instead some alternative representation with the interpretation of lower and upper probabilities.

An alternative to the measure ofimprecision used in the present paper, and hence relevant for future work,is the Hartley-like measure of non-specificity, variants of which exist for both possibility and evidence theory; see Klir (2006; 1999). 
Further work in relation to the suggested measure could also be directed towards implementation of the type II measure on more complex systems. Moreover, possibility theory provides a relatively simple and hence convenient uncertainty representation to use for the implementation of the suggested measures; however, other representations couldalso be considered in terms of application depending on the particular uncertainty setting. Finally, future work will also be devoted to the investigation of the proposed imprecision uncertainty importance measures in presence of dependences in the input considered for the analysis.

\section{Acknowledgements}

The authors are grateful to two anonymous reviewers for useful comments and suggestions to an earlier version of the present paper.

The work of T. Aven and R. Flage has been partially funded by The Research Council of Norway through the PETROMAKS research program. The financial support is gratefully acknowledged.

The work of E. Zio and P. Baraldi has been partially funded by the "Foundation pour une Culture de Securite' Industrielle" of Toulouse, France, under the research contract AO200904.

\section{References}

Apostolakis, G.E. 1990. The concept of probability in safety assessments of technological systems.Science 250(4986): 1359-1364.

Aven, T. \& Jensen, U. 1999.Stochastic Models in Reliability. New York: Springer.

Aven, T. \&Nøkland, T.E. 2010 On the use of uncertainty importance measures in reliability and risk analysis. Reliability Engineering and System Safety 95(2): 127-133. 
Baraldi, P., Librizzi, M., Zio, E., Podofillini, L. \&Dang, V.N. 2009. Two techniques of sensitivity and uncertainty analysis of fuzzy expert systems. Expert Systems with Applications 36(10): 12461-12471.

Baraldi, P. \&Zio, E. 2008 A combined Monte Carlo and possibilistic approach to uncertainty propagation in event tree analysis.Risk Analysis 28(5): 1309-1325.

Baudrit, C., Dubois D. \&Guyonnet D. 2006.Joint propagation of probabilistic and possibilistic information in risk assessment.IEEE Transactions on Fuzzy Systems 14(5): 593-608.

Berger, J.O. 1984 The robust Bayesian viewpoint. In J. B. Kadane, JB (ed.), Robustness of Bayesian Analyses: 63-144. Amsterdam: Elsevier Science.

Borgonovo, E. 2006.Measuring uncertainty importance: Investigation and comparison of alternative approaches.Risk Analysis 26(5): 1349-1361.

Dempster, A.P. (1967) Upper and lower probabilities induced by a multivalued mapping. The Annals of Mathematical Statistics 38: 325-339.

Dubois, D. 2006. Possibility theory and statistical reasoning. Computational Statistics \& Data Analysis 51: 47-69.

Dubois, D. \&Prade, H. (1992)When upper probabilities are possibility measures. Fuzzy Sets and Systems 49: 65-74.

Dubois, D., Prade H. \&Sandri S. 1993. On possibility/probability transformations. In: Lowen R, Roubens M, editors. Fuzzy Logic: State of the Art. Dordrecht: Kluwer Academic Publishers. pp. 103-112.

Cheok, M.C., Parry, G.W. and Sherry, R.R. 1998. Use of importance measures in riskinformed regulatory applications.Reliability Engineering and System Safety 60: 213-226.

Dubois, D. \&Prade, H. 1988.Possibility Theory - An Approach to Computerized Processing of Uncertainty. New York: Plenum Press. 
Ferson, S., Kreinovich, V., Ginzburg, L., Myers, D.S. \&Sentz, K. 2003.Constructing probability boxes and Dempster-Shafer structures.Technical Report SAND2002-4015, Sandia National Laboratories.

Flage, R., Baraldi, P., Zio, E.\& Aven, T. (2011) On imprecision in relation to uncertainty importance measures. In: Bérenguer, C., Grall, A.\&GuedesSoares, C. (eds) Advances in Safety, Reliability and Risk Management. Proceedings of the European Safety and Reliability Conference (ESREL) 2011, Troyes, France, 18-22 September 2011. pp. 2250-2255.

Helton, J C \&Burmaster, D E (1996) Guest editorial: treatment of aleatory and epistemic uncertainty in performance assessments for complex systems. Reliability Engineering and System Safety 54: 91-94.

Iman, R.L. 1987. A matrix-based approach to uncertainty and sensitivity analysis for fault trees. Risk Analysis 7(1): 21-33.

Kalos M.H. \& Whitlock P.A. 1986.Monte Carlo Methods. Volume I: Basics. Wiley.

Kaplan, S. \& Garrick, B.J. 1981.On the quantitative definition of risk.Risk Analysis 1(1): 1127.

Klir, G.J. (2006) Uncertainty and Information: Foundations of Generalized Information Theory. Hoboken, N.J.: Wiley-Interscience.

Klir, G.J. (1999) Uncertainty and Information Measures for ImpreciseProbabilities: An Overview. In: De Cooman, G.,Cozman, F.G., Moral, S. \&Walley, P. (eds)ISIPTA '99.Proceedings of the First International Symposium on Imprecise Probabilities and Their Applications, Ghent, Belgium, 29 June - 2 July 1999. pp. 234-240.

Pedroni, N. \& Zio, E. 2012. Empirical comparison of methods for the hierarchical propagation of hybrid uncertainty in risk assessment in presence of dependences, International Journal of Uncertainty, Fuzziness and Knowledge-Based Systems. 20(4):509-557. 
Rausand, M. \&Høyland, A. 2004. System Reliability Theory: Models, Statistical Methods, and Applications. 2nd ed. Hoboken, N.J.: Wiley-Interscience.

Shafer, G. 1976. A Mathematical Theory of Evidence.Princeton University Press.

Suresh, P. V., Babar, A. K., \& Raj, V. V. (1996). Uncertainty in fault tree analysis: A fuzzy approach. Fuzzy Sets and Systems, 83, 135-141.

Walley, P. 1991. Statistical Reasoning with Imprecise Probabilities. London: Chapman and Hall.

Weichselberger, K. 2000. The theory of interval probability as a unifying concept for uncertainty.International Journal of Approximate Reasoning 24: 149-170.

Zadeh L.A. 1965. Fuzzy sets. Information and Control 8: 338-353.

Zio, E. 2009.Computational Methods for Reliability and Risk Analysis. Hackensack, N.J.: World Scientific.

Liping, H. \& Fuzheng, Q. 2009. Possibilistic entropy-based measure of importance in fault tree analysis. Journal of Systems Engineering and Electronics 20(2): 434-444. 